\title{
Existence and multiplicity results for nonlinear critical Neumann problem on compact Riemannian manifolds
}

\author{
Youssef Maliki
}

\begin{abstract}
In this work, we study on a compact Riemannian manifold with boundary, the problems of existence and multiplicity of solutions to a Neumann problem involving the $p$-Laplacian operator and critical Sobolev exponents.
\end{abstract}

Mathematics Subject Classification (2000). 58J05.

Keywords. Riemannian manifolds, Neumann problems, $p$-Laplacian operator, Critical exponents.

\section{Introduction}

Let $(\bar{M}, g)$ be an $n(n \geq 3)$-dimensional Riemannian manifolds with interior $M$ and boundary $\partial M$. In this paper, we are interested in the problem of finding positive solutions on $\bar{M}$ to the nonlinear Neumann boundary value problem

$$
\begin{cases}\Delta_{p} u+a(x)|u|^{p-2} u=f(x)|u|^{p^{*}-2} u & \text { in } M \\ \left|\nabla_{g} u\right|^{p-2} \partial_{\nu_{g}} u+k(x)|u|^{p-2} u=K(x)|u|^{p^{* *}-2} u & \text { on } \partial M\end{cases}
$$

where $\partial_{\nu_{g}}$ is the outer unit normal derivative, $p \in(1, n), \Delta_{p}=-\operatorname{div}\left(\left|\nabla_{g} u\right|^{p-2}\right.$ $\left.\nabla_{g} u\right)$ is the $p$-Laplacian operator, $p^{*}=\frac{n p}{n-p}$ and $p^{* *}=\frac{(n-1) p}{n-p}$.

Problem (1.1) in case $p=2$ appears in differential geometry as the problem of prescribing scalar and mean curvatures, that is, existence of a positive solution of such problem provides the manifold $\bar{M}$ with a conformal metric to $g$ with scalar curvature the function $f$ in $M$ and mean curvature the function $K$ on the boundary $\partial M$. Anyway, this problem was widely studied, we cite for instance $[22,23],[3,4,15,20]$.

In the case $p \neq 2$, though the geometric aspect of problem (1.1) is vague, study of such problem in the Riemmannian context has drawn the attention of some authors. For example, in case of equation (1.1) without boundary 
condition, some existence and multiplicity results on compact and complete manifolds have been obtained by $[6,8-11,13,21]$.

Recently, in [18], using the variational method, the authors proved an existence result for problem (1.1) on solid torus in $\mathbb{R}^{3}$ under the conditions that either the function $f$ is positive and $K$ is arbitrary or $f$ is nonnegative and $K$ is positive.

Successively, in [19] they obtained a similar result on general compact Riemannian manifolds under the same conditions.

Independently, in [24] the authors dealt with problem (1.1) in the case where the function $f$ changes sing and the function $K$ is non positive. They obtained an existence result for the critical case and multiplicity result for the subcritical case. They used a fibering method introduced by Pohozaev [26].

In the present work, we are interested in the case where both functions $f$ and $K$ change sign on $\bar{M}$. This situation, missing in [24], is more complicated and application of direct variational techniques is inadequate contrariwise to the cases treated in $[15,18,19]$. We should also point out that arguments used in [24] are inefficient.

In [27], the author considered for $p=2$ and $f$ changes sign the problem (1.1) without boundary condition. He introduced some variational techniques and derived existence of a solution in the critical case and a pair of solutions in the subcritical case. These techniques rely on considering minimization problem on suitable sets from which one can construct a sequence of curves satisfying a certain geometry that allows to get pair of solutions in the subcritical case. In our case, the functions involved in the right hand sides both change sign, this permits adaptation ideas in [27] and thereby multiplicity of solutions in the subcritical case can be obtained. Under further conditions and by using a concentration-compactness lemma for manifolds with boundary [12], we prove existence of a pair of solutions to the critical case which includes the geometric case of prescribing scalar and mean curvatures. We assert by the way the existence of a pair of conformal metrics that share the same indefinite sign scalar and mean curvatures.

Note that the techniques in [27] have been adapted for equation (1.1) without boundary condition in [10] and for a $Q$-curvature equation in [7].

\section{Notations}

Along all this paper we denote by,

1. $a$ and $k$ two negative constants,

2. $f$ and $K$ two changing functions respectively on $M$ and $\partial M$,

3. $q$ and $r$ two constants such that $1<p<q \leq p^{*}, 1<p<r \leq p^{* *}$ and $p r<p q<r^{2}$,

4. $\|u\|_{p, M},\|u\|_{p, \partial M}$ the $L_{p}$ norms respectively on $M$ and $\partial M$,

5. $H_{1}^{p}(M)$ the Sobolev space of the functions in $L_{p}$ with gradient in $L_{p}$,

6. $K_{1}, K_{2}$ the best constants defined in the Sobolev and trace Sobolev inequalities which are the best constants such that there exist positive 
constants $A$ and $B$ such that

$$
\begin{aligned}
\|u\|_{q, M}^{p} & \leq K_{1}\left\|\nabla_{g} u\right\|_{p, M}^{p}+A\|u\|_{p, M}^{p}, p<q \leq p^{*} \\
\|u\|_{r, \partial M}^{p} & \leq K_{2}\left\|\nabla_{g} u\right\|_{p, M}^{p}+B\|u\|_{p, \partial M}^{p}, p<r \leq p^{* *}
\end{aligned}
$$

7. $h^{-}=\min (h, 0), h^{+}=\max (h, 0)$, respectively the negative and positive part of a function $h$.

By a solution of problem (1.1), we mean a function $u \in H_{1}^{p}(M)$ such that for every $v \in \mathcal{C}^{\infty}(\bar{M})$ we have

$$
\begin{gathered}
\int_{M}\left|\nabla_{g} u\right|^{p-2} g\left(\nabla_{g} u, \nabla_{g} v\right) d v_{g}+\int_{M} a|u|^{p-2} u v d v_{g}+\int_{\partial M} k|u|^{p-2} u v d \sigma_{g} \\
=\int_{M} f|u|^{p^{*}-2} u v d v_{g}+\int_{\partial M} K|u|^{p^{* *}-2} u v d \sigma_{g} .
\end{gathered}
$$

By regularity results [25], we get that $u \in \mathcal{C}^{1, \beta}(\bar{M})$, for some $\beta \in(0,1)$.

\section{Statement of the results}

Our purpose is to prove existence and multiplicity of solutions to problem (1.1), it is to seek critical points $u \in H_{1}^{p}(M)$ of the energy functional

$$
\begin{aligned}
E(u)= & \int_{M}\left|\nabla_{g} u\right|^{p} d v_{g}+a \int_{M}|u|^{p} d v_{g}+k \int_{\partial M}|u|^{p} d \sigma_{g} \\
& -\frac{n-p}{n} \int_{M} f|u|^{p^{*}} d v_{g}-\frac{n-p}{n-1} \int_{\partial M} K|u|^{p^{* *}} d \sigma_{g} .
\end{aligned}
$$

Define the quantity

$$
\lambda_{f, K}=\inf _{\mathcal{A}} \frac{\left\|\nabla_{g} u\right\|_{p, M}^{p}}{|a|\|u\|_{p, M}^{p}+|k|\|u\|_{p, \partial M}^{p}}
$$

where

$$
\mathcal{A}=\left\{u \in H_{1}^{p}(M), u \geq 0: \int_{M}\left|f^{-}\right| u d v_{g}+\int_{\partial M}\left|K^{-}\right| u d \sigma_{g}=0\right\}
$$

In this paper, we prove the following theorems

Theorem 3.1. Let $(\bar{M}, g)$ be a compact Riemannian manifold with interior $M$ and boundary $\partial M$.

There exists a positive constant $C$ such that if the functions $f$ and $K$ satisfies the following conditions

1. $\lambda_{f, K}>1$,

2. $\sup _{M} f>0, \sup _{\partial M} K>0$ and $\sup _{M} f+\sup _{\partial M} K \leq C$,

then problem (1.1) admits a positive solution.

The following theorem concerns the multiplicity of the problem

$$
\begin{cases}\Delta_{p} u+a(x)|u|^{p-2} u=f(x)|u|^{q-2} u & \text { in } M ; \\ \left|\nabla_{g} u\right|^{p-2} \partial_{\nu_{g}} u+k(x)|u|^{p-2} u=K(x)|u|^{r-2} u & \text { on } \partial M .\end{cases}
$$

where $p<q<p^{*}, p<r<p^{* *}$ and $r<q<\frac{r^{2}}{p}$. 
Theorem 3.2. Let $(\bar{M}, g)$ be a compact Riemannian manifold with interior $M$ and boundary $\partial M$. There exists a positive constants $C_{q, r}$ such that if the functions $f$ and $K$ satisfy the following conditions

1. $\lambda_{f, K}>1$,

2. $\sup _{M} f>0, \sup _{\partial M} K>0$ and $\sup _{M} f+\sup _{\partial M} K \leq C_{q, r}$,

then problem (3.1) admits at least two distinct positive solutions.

Under further conditions, we prove the multiplicity of problem (1.1). Let $u$ be the solution given by Theorem 3.1 and $C_{q, r}$ the constant given in Theorem 3.2.

Denote $I$ the functional

$$
I(u)=\int_{M}\left|\nabla_{g} u\right|^{p} d v_{g}+a \int_{M}|u|^{p} d v_{g}+k \int_{\partial M}|u|^{p} d \sigma_{g} .
$$

We prove the following theorem

Theorem 3.3. Let $(\bar{M}, g)$ be a compact Riemannian manifold with interior $M$ and boundary $\partial M$. There exists a positive constant $C$ such that if the functions $f$ and $K$ satisfy the following conditions

1. $\lambda_{f, K}>1$,

2. $\sup _{M} f>0, \sup _{\partial M} K>0$ and $\sup _{M} f+\sup _{\partial M} K \leq C$,

3. $\frac{p(n-1)}{n(p-1)}\left(\sup _{M} f\right)^{-\frac{n-p}{p}}\left(\sup _{\partial M} K\right)^{\frac{n-p}{p-1}} K_{1}^{-n} K_{2}^{\frac{p(n-1)}{p-1}} \leq 1$,

and that there exists a positive function $\Phi \in H_{1}^{p}(M)$ such that $I(\Phi)>$ $0, \int_{M} f \Phi^{p^{*}} d v_{g}>0, \int_{\partial M} K \Phi^{p^{* *}} d \sigma_{g}>0$ and

$$
0<\sup _{\lambda \in[0,1]} E(\lambda \Phi)<E(u)+\frac{p}{n}\left[\sup _{M} f\right]^{1-\frac{n}{p}} K_{1}^{-\frac{n}{p}}
$$

then, problem (1.1) admits at least two distinct positive solutions.

\section{Necessary condition}

Recall that we have defined $\lambda_{f, K}$ as

$$
\lambda_{f, K}=\inf _{\mathcal{A}} \frac{\left\|\nabla_{g} u\right\|_{p, M}^{p}}{|a|\|u\|_{p, M}^{p}+|k|\|u\|_{p, \partial M}^{p}},
$$

where

$$
\mathcal{A}=\left\{u \in H_{1}^{p}(M), u \geq 0: \int_{M}\left|f^{-}\right| u d v_{g}+\int_{\partial M}\left|K^{-}\right| u d \sigma_{g}=0\right\} .
$$

If $\mathcal{A}=\phi$, set $\lambda_{f, K}=\infty$.

Let us prove the following lemma

Lemma 4.1. If the problem 1.1 admits a solution, then $\lambda_{f, K} \geq 1$.

Proof. First, we show that $\lambda_{f, K}$ is attained. By homogeneity, we may take $\left\{u_{i}\right\} \subset H_{1}^{p}(M), u_{i} \geq 0$ a minimizing sequence for $\lambda_{f, K}$ such that $\left\|u_{i}\right\|_{p, M}^{p}+$ $\left\|u_{i}\right\|_{p, \partial M}^{p}=1$, then $u_{i}$ is bounded in $H_{1}^{p}(M)$. By the Rellich-Kondrakov and 
Banach theorems, there exist a subsequence $u_{i}$ and a function $u$ such that $u_{i}$ converges weakly in $H_{1}^{p}(M)$, strongly in $L_{s}(M)$ and $L_{t}(\partial M), s<p^{*}, t<$ $p^{* *}$, almost everywhere in $M$ and in the sense of trace on $\partial M$. Then we get $\|u\|_{p, M}^{p}+\|u\|_{p, \partial M}^{p}=1$ and $u \in \mathcal{A}$. Moreover, the weak convergence gives that

$$
\left\|\nabla_{g} u\right\|_{p, M}^{p} \leq \liminf \left\|\nabla_{g} u_{i}\right\|_{p, M}^{p}
$$

thus

$$
\lambda_{f, K}=\frac{\left\|\nabla_{g} u\right\|_{p, M}^{p}}{|a|\|u\|_{p, M}^{p}+|k|\|u\|_{p, \partial M}^{p}} .
$$

which means that $\lambda_{f, K}$ is attained by the function $u$ and by regularity theorems $u$ is $\mathcal{C}^{1, \alpha}(M)$ for certain $\alpha \in(0,1)$.

Now, we state the following generalized Picone's inequality [1]: for two differentiable functions $u \geq 0$ and $v>0$, we have

$$
\left|\nabla_{g} u\right|^{p} \geq\left|\nabla_{g} v\right|^{p-2} g\left(\nabla_{g} v, \nabla_{g} \frac{u^{p}}{v^{p-1}}\right) .
$$

Take $v \geq 0$ a minimizer for $\lambda_{f, K}$ and let $u>0$ be a positive solution of the problem (1.1), then we have

$$
\begin{aligned}
& \int_{M}\left|\nabla_{g} u\right|^{p-2} g\left(\nabla_{g} u, \nabla_{g} \frac{v^{p}}{u^{p-1}}\right) d v_{g}=|a|\|v\|_{p, M}+|k|\|v\|_{p, \partial M} \\
& +\int_{M} f^{+} u^{p^{*}-p} v^{p} d v_{g}+\int_{\partial M} K^{+} u^{p^{* *}-p} v^{p} d v \sigma_{g} \\
& -\int_{M}\left|f^{-}\right| u^{p^{*}-p} v^{p} d v_{g}-\int_{\partial M}\left|K^{-}\right| u^{p^{* *}-p} v^{p} d v \sigma_{g},
\end{aligned}
$$

since for $p>1$, the supports of the functions $u^{p^{*}-p} v^{p}$ and $u^{p^{* *}-p} v^{p}$ are included respectively in the regions where $f(x)>0$ and $K(x)>0$, that is $\int_{M}|f|^{-} u^{p^{*}-p} v^{p} d v_{g}+\int_{\partial M}|K|^{-} u^{p^{* *}-p} v^{p} d v \sigma_{g}=0$, by (4.1) and (4.2) we get

$$
\lambda_{f, K}=\frac{\left\|\nabla_{g} v\right\|_{p, M}}{|a|\|v\|_{p, M}+|k|\|v\|_{p, \partial M}} \geq 1 .
$$

\section{Subcritical problem: multiplicity result}

In this section, we prove existence of at least two positive solutions of problem 3.1 , we look for critical points of the following functional

$$
\begin{aligned}
E_{q, r}(u)= & \int_{M}\left|\nabla_{g} u\right|^{p} d v_{g}+a \int_{M}|u|^{p} d v_{g}+k \int_{\partial M}|u|^{p} d \sigma_{g} \\
& \left.\left.\left.\left.-\frac{p}{q} \int_{M} f|u|^{q} d v_{g}-\frac{p}{r} \int_{\partial M} K|u|^{r} d \sigma_{g}, q \in\right] p, p^{*}\right], r \in\right] p, p^{* *}\right],
\end{aligned}
$$

which is bounded on the set

$$
\mathcal{S}_{\ell, q, r}=\left\{u \in H_{1}^{p}(M):\|u\|_{q, M}^{q}+\|u\|_{r, \partial M}^{r}=\ell\right\},
$$


set

$$
\mu_{\ell, q, r}=\inf _{u \in \mathcal{S}_{\ell, q, r}} E_{q, r}(u) .
$$

We prove the following lemmas

Lemma 5.1. $\mu_{\ell, q, r}$ is attained for $q<p^{*}$ and $r<p^{* *}$.

Proof. Let $\ell>0$ and $\left\{u_{i}\right\}_{i \geq 1} \subset \mathcal{S}_{\ell, q, r}$ be a minimizing sequence, that is $\left\|u_{i}\right\|_{q, M}^{q}+\left\|u_{i}\right\|_{r, \partial M}^{r}=\ell$ and $\lim _{i \rightarrow \infty} E_{q, r}\left(u_{i}\right)=\mu_{\ell, q, r}$. For $i$ large enough we can assume $E_{q, r}\left(u_{i}\right) \leq \mu_{\ell, q, r}+1$, which gives that

$$
\begin{aligned}
& \left\|\nabla_{g} u_{i}\right\|_{p, M}^{p} \leq \mu_{\ell, q, r}+|a| \operatorname{Vol}(M)^{1-\frac{p}{q} \ell^{\frac{p}{q}}}+|k| \operatorname{Vol}(\partial M)^{1-\frac{p}{r}} \ell^{\frac{p}{r}} \\
& \quad+\left(\sup _{M} f+\sup _{\partial M} K\right) \ell+1 .
\end{aligned}
$$

Thus, the sequence $\left\{u_{i}\right\}_{i \geq 1}$ is bounded in $H_{1}^{p}(M)$. Since the inclusions of $H_{1}^{p}(M)$ in $L_{p}(M), L_{p}(\partial M), L_{q}(M)$ and $L_{r}(\partial M)$ are compact for $q<p^{*}$ and $r<p^{* *}$, then there exist a subsequence $u_{i}$ and a function $u \in H_{1}^{p}(M)$ such that $u_{i}$ converges to $u$ weakly in $H_{1}^{p}(M)$ and strongly in each of the spaces $L_{p}(M), L_{p}(\partial M), L_{q}(M), L_{r}(\partial M)$. It converges also to $u$ almost everywhere in $M$ and in sense of trace on $\partial M$. Thus, $u \in \mathcal{S}_{\ell, q, r}$ and $E_{q, r}(u) \geq \mu_{\ell, q, r}$. Moreover, the strong and weak convergence imply that

$$
\left\|\nabla_{g} u\right\|_{M, p} \leq \liminf \left\|\nabla_{g} u_{i}\right\|_{M, p}
$$

hence

$$
E_{q, r}(u) \leq \lim _{i \rightarrow \infty} E_{q, r}\left(u_{i}\right)=\mu_{\ell, q, r}
$$

which means that $\mu_{\ell, q, r}$ is attained.

Lemma 5.2. $\mu_{\ell, q, r}$ is continuous as a function in the variable $\ell$.

Proof. Let $\ell \in] 0, \infty\left[\right.$ and $\left.\left\{\ell_{n}\right\}_{n \in \mathbb{N}} \subset\right] 0, \infty[$ be a sequence such that $\lim _{n \rightarrow \infty} \ell_{n}=\ell<\infty$. By Lemma 5.1, for every $n \in \mathbb{N}$ there exist $u_{n} \in$ $\mathcal{S}_{\ell_{n}, q, r}$ such that $E_{q, r}\left(u_{n}\right)=\mu_{\ell_{n}, q, r}$ and there exists a function $u \in \mathcal{S}_{\ell, q, r}$ such that $E_{q, r}(u)=\mu_{\ell, q, r}$. The sequence $u_{n}$ is bounded in the spaces $L_{q}(M), L_{r}(\partial M), L_{p}(M), L_{p}(\partial M)$ and satisfies

$$
\begin{aligned}
& \left\|\nabla_{g} u_{n}\right\|_{p, M}^{p} \leq \mu_{\ell_{n}, q, r}+|a| \operatorname{vol}(M)^{1-\frac{p}{q}} \ell_{n}^{\frac{p}{q}}+|k| \operatorname{vol}(\partial M)^{1-\frac{p}{r}} \ell_{n}^{\frac{p}{r}} \\
& \quad+\left(\sup _{M} f+\sup _{\partial M} K\right) \ell_{n} .
\end{aligned}
$$

On the other hand, there exits $t_{n}>0, \lim _{n \rightarrow \infty} t_{n}=1$ such that $t_{n} u \in \mathcal{S}_{\ell_{n}, q, r}$. Then

$$
\mu_{\ell_{n}, q, r} \leq t_{n}{ }^{p}\left\|\nabla_{g} u\right\|_{p, M}^{p}+\left(t_{n}^{q}\left|\inf _{M} f\right|+t_{n}^{r}\left|\inf _{\partial M} K\right|\right) \ell,
$$

which gives that the sequence $\left\{u_{n}\right\}_{n \geq 0}$ is bounded in $H_{1}^{p}(M)$. Up to a subsequence $u_{n}$ converges to a function $\hat{u}$ strongly in the spaces $L_{q}(M), L_{r}(\partial M)$, $L_{p}(M), L_{p}(\partial M)$ and weakly in $H_{1}^{p}(M)$. Thus $\hat{u} \in \mathcal{S}_{\ell, q, r}$ which implies that $E_{q, r}(\hat{u}) \geq E_{q, r}(u)$. 
Hence,

$$
\lim _{n \rightarrow \infty} \inf E_{q, r}\left(u_{n}\right)-E_{q, r}(u) \geq 0 .
$$

On the other hand, we have

$$
\begin{aligned}
E_{q, r}\left(u_{n}\right) \leq & t_{n}^{p}\left(\left\|\nabla_{g} u\right\|_{p, M}^{p}+a\|u\|_{p, M}^{p}+k\|u\|_{p, \partial M}^{p}\right) \\
& -t_{n}^{q} \frac{p}{q} \int_{M} f|u|^{q} d v_{g}-t_{n} \frac{p}{r} \int_{\partial M} K|u|^{r} d \sigma_{g},
\end{aligned}
$$

thus

$$
\lim _{n \rightarrow \infty} \sup E_{q, r}\left(u_{n}\right)-E(u)_{q, r} \leq 0 .
$$

Therefore, $\mu_{\ell, q, r}$ is continuous.

Lemma 5.3. $\mu_{\ell, q, r}$ is negative for $l$ small.

Proof. For $\ell>0$, let $m_{\ell}>0$ be the solution of the equation $\operatorname{vol}(M) m_{\ell}^{q}+$ $\operatorname{vol}(\partial M) m_{\ell}^{r}=\ell$. Take the constant function $u=m_{\ell}$, then $u \in \mathcal{S}_{\ell, q, r}$ and

$$
\begin{aligned}
\mu_{\ell, q, r} \leq E_{q, r}(u)= & m_{\ell}^{p}\left[-|a| \operatorname{vol}(M)-|k| \operatorname{vol}(\partial M)-\frac{p}{q} m_{\ell}^{q-p} \int_{M} f d v_{g}\right. \\
& \left.-\frac{p}{r} m_{\ell}^{r-p} \int_{\partial M} K d \sigma_{g}\right],
\end{aligned}
$$

If $\ell$ is small, then so is $m_{\ell}$ and we conclude the lemma.

Lemma 5.4. $\mu_{\ell, q, r}$ is negative for $\ell$ large.

Proof. Let $u$ and $v$ be respectively smooth functions defined on $M$ and $\partial M$ with supports included respectively in the sets where $f(x)>0$ and $K(x)>0$ and such that $\|u\|_{q, M}^{q}=1$ and $\|v\|_{r, \partial M}^{r}=1$. For $x \in \bar{M}$, set $h(x)=\frac{1}{2}\left(\ell^{\frac{1}{q}} u(x)+\ell^{\frac{1}{r}} v(x)\right)$, then $h \in \mathcal{S}_{\ell, q, r}$ and we get

$$
\begin{aligned}
\mu_{\ell, q, r} \leq E(h)=\frac{1}{2^{p}} \ell & {\left[\left(\left\|\nabla_{g} u\right\|_{p, M}^{p}+a\|u\|_{p, M}^{p}\right) \ell^{\frac{p}{q}-1}+k\|u\|_{p, \partial M}^{p} \ell^{\frac{p}{r}-1}\right.} \\
& \left.-\frac{1}{2^{q-p}} \int_{M} f|u|^{q} d v_{g}-\frac{1}{2^{r-p}} \int_{\partial M} K|v|^{r} d \sigma_{g}\right] .
\end{aligned}
$$

since $\int_{M} f|u|^{q} d v_{g}>0$ and $\int_{\partial M} K|v|^{r} d \sigma_{g}>0$, then for $\ell$ large enough $\mu_{\ell, q, r}$ $<0$.

Now, let us define the quantity

$$
\lambda_{f, K, \eta, q, r}=\inf _{u \in \mathcal{K}(\eta, q, r)} \frac{\left\|\nabla_{g} u\right\|_{p, M}^{p}}{|a|\|u\|_{p, M}^{p}+|k|\|u\|_{p, \partial M}^{p}}
$$

where

$$
\begin{aligned}
& \mathcal{K}(\eta, q, r)=\left\{u \in H_{1}^{p}(M):\|u\|_{q, M}^{q}+\|u\|_{r, \partial M}^{r}=1\right. \text { and } \\
& \quad \frac{p}{q} \int_{M}\left|f^{-}\left\|\left.u\right|^{q} d v_{g}+\frac{p}{r} \int_{\partial M}\left|K^{-} \| u\right|^{r} d \sigma_{g} \leq \eta\left(\int_{M}\left|f^{-}\right| d v_{g}+\int_{\partial M}\left|K^{-}\right| d \sigma_{g}\right)\right\} .\right.
\end{aligned}
$$


Lemma 5.5. For $(q, r) \in] p, p^{*}[\times] p, p^{* *}\left[, \lambda_{f, K, \eta, q, r}\right.$ converges to $\lambda_{f, K}$ when $\eta$ goes to zero.

Proof. First let us prove that $\lambda_{f, K, \eta, q, r}$ is attained. Let $u_{i, \eta} \in \mathcal{K}(\eta, q, r)$ be a minimizing sequence for $\lambda_{f, K, \eta, q, r}$, then for $i$ large enough we can have

$$
\left\|\nabla_{g} u_{i, \eta}\right\|_{p, M}^{p} \leq \lambda_{f, K, \eta, q, r}\left(|a|\left\|u_{i, \eta}\right\|_{p, M}^{p}+|k|\left\|u_{i, \eta}\right\|_{p, \partial M}^{p}\right)+1 .
$$

Hence, the sequence $u_{i, \eta} \in \mathcal{K}(\eta, q, r)$ is bounded in $H_{1}^{p}(M)$ and up to a subsequence $u_{i, \eta}$ converges to a function $u_{\eta}$ weakly in $H_{1}^{p}(M)$ and strongly in each of the spaces $L_{p}(M), L_{p}(\partial M), L_{q}(M), L_{r}(\partial M)$. Thus, $u_{\eta} \in \mathcal{K}(\eta, q, r)$. Moreover,

$$
\left\|\nabla_{g} u_{\eta}\right\|_{M, p} \leq \lim _{i \rightarrow \infty} \inf \left\|\nabla_{g} u_{i, \eta}\right\|_{M, p}
$$

Hence

$$
\frac{\left\|\nabla_{g} u_{\eta}\right\|_{M, p}}{|a|\left\|u_{\eta}\right\|_{p, M}^{p}+|k|\left\|u_{\eta}\right\|_{p, \partial M}^{p}}=\lambda_{f, K, \eta, q, r}
$$

Now, consider $u_{\eta}$ a sequence of $\eta$. First we observe that if $u \in \mathcal{A}$ then there exists $\beta>0$ such that $\beta u \in \mathcal{K}(\eta, q, r)$. By homogeneity, we get then that the sequence $\lambda_{f, K, \eta, q, r}$ is bounded by $\lambda_{f, K}$. Thus, the sequence $u_{\eta}$ is bounded in $H_{1}^{p}(M)$ and there exists a subsequence that converges, when $\eta$ goes to zero, to a function $u$ weakly in $H_{1}^{p}(M)$ and strongly in $L_{p}(M), L_{p}(\partial M), L_{q}(M)$ and $L_{r}(\partial M)$. Hence, $u \in \mathcal{A}$. On the other hand

$$
\left\|\nabla_{g} u\right\|_{p, M}^{p} \leq \lim _{\eta \rightarrow 0} \inf \left\|\nabla_{g} u_{\eta}\right\|_{p, M}^{p} .
$$

Thus

$$
\lambda_{f, K} \leq \frac{\left\|\nabla_{g} u\right\|_{p, M}^{p}}{|a|\|u\|_{p, M}^{p}+|k|\|u\|_{p, \partial M}^{p}} \leq \lim _{\eta \rightarrow 0} \inf \lambda_{f, K, \eta, q, r} .
$$

Now, coming back to the function $\ell \in(0, \infty) \longrightarrow \mu_{\ell, q, r}$ to prove the following lemma

Lemma 5.6. Suppose that $\lambda_{f, K}>1$. There exists a positive constant $C_{q, r}$ such that if $\sup _{M} f+\sup _{\partial M} K \leq C_{q, r}$ then there exists an interval $\left[\ell_{1}, \ell_{2}\right]$ such that $\mu_{\ell, q, r}$ is positive for every $\ell \in\left[\ell_{1}, \ell_{2}\right]$.

Proof. First, if $\lambda_{f, K}>1$, it follows from Lemma 5.5 that for $\eta$ small enough $\lambda_{f, K, \eta, q, r}-1>0$.

Let $u \in H_{1}^{p}(M)$ be the function given by Lemma(5.1) such that $\|u\|_{q, M}^{q}+$ $\|u\|_{r, \partial M}^{r}=\ell$ and $E_{q, r}(u)=\mu_{\ell, q, r}$ with

$$
\ell \geq \ell_{q, r}=\max \left(\left(\frac{1+|a| \operatorname{vol}(M)^{1-\frac{p}{q}}}{\eta \int_{M}\left|f^{-}\right| d v_{g}}\right)^{\frac{q}{r-p}},\left(\frac{1+|k| \operatorname{vol}(\partial M)^{1-\frac{p}{r}}}{\eta \int_{\partial M}\left|K^{-}\right| d \sigma_{g}}\right)^{\frac{q r}{r^{2}-p q}}\right)
$$

take $\eta>0$ small enough so that $\ell>1$. 
Denote by $G_{q, r}$ the functional

$$
\begin{aligned}
G_{q, r}(u)= & \left\|\nabla_{g} u\right\|_{p, M}^{p}+a\|u\|_{p, M}^{p}+k\|u\|_{p, \partial M}^{p} \\
& +\frac{p}{q} \int_{M}\left|f ^ { - } \left\|\left.u\right|^{q} d v_{g}+\frac{p}{r} \int_{\partial M}\left|K^{-} \| u\right|^{r} d \sigma_{g} .\right.\right.
\end{aligned}
$$

On one hand, if

$$
\frac{p}{q} \int_{M}\left|f^{-}\right||u|^{q} d v_{g}+\frac{p}{r} \int_{\partial M}\left|K^{-}\right||u|^{r} d \sigma_{g} \geq \eta \ell^{\frac{r}{q}}\left(\int_{M}\left|f^{-}\right| d v_{g}+\int_{\partial M}\left|K^{-}\right| d \sigma_{g}\right),
$$

then, by Hölder inequality we get

$$
\begin{aligned}
G_{q, r}(u) \geq & \operatorname{vol}(M)^{1-\frac{p}{q}} a \ell^{\frac{p}{q}}+\operatorname{vol}(\partial M)^{1-\frac{p}{r}} k \ell^{\frac{p}{r}} \\
& +\ell^{\frac{r}{q}} \eta\left(\int_{M}\left|f^{-}\right| d v_{g}+\int_{\partial M}\left|K^{-}\right| d \sigma_{g}\right) \\
\geq & {\left[\eta \int_{M}\left|f^{-}\right| d v_{g} \ell^{\frac{r-p}{q}}-\operatorname{vol}(M)^{1-\frac{p}{q}}|a|\right] \ell^{\frac{p}{q}} } \\
& +\left[\eta \int_{\partial M}\left|K^{-}\right| d \sigma_{g} \ell^{\frac{r^{2}-p q}{q r}}-\operatorname{vol}(\partial M)^{1-\frac{p}{r}}|k|\right] \ell^{\frac{p}{r}},
\end{aligned}
$$

using (5.1), we get

$$
G_{q, r}(u) \geq \ell^{\frac{p}{q}}+\ell^{\frac{p}{r}} .
$$

On the other hand, if

$$
\begin{aligned}
& \frac{p}{q} \int_{M}\left|f^{-}\right||u|^{q} d v_{g}+\frac{p}{r} \int_{\partial M}\left|K^{-}\right||u|^{r} d \sigma_{g} \\
& \quad \leq \ell^{\frac{r}{q}} \eta\left(\int_{M}\left|f^{-}\right| d v_{g}+\int_{\partial M}\left|K^{-}\right| d \sigma_{g}\right),
\end{aligned}
$$

let $\delta>0$ be a solution of the equation $\delta^{q}\|u\|_{q, M}^{q}+\delta^{r}\|u\|_{r, M}^{r}=1$, it can be easily seen that $\ell^{-\frac{1}{r}}<\delta<\ell^{-\frac{1}{q}}$. This implies together with (5.2) that $\delta u \in \mathcal{K}(\eta, q, r)$, we get then,

$$
\left\|\nabla_{g} u\right\|_{p, M}^{p} \geq \lambda_{f, K, \eta, q, r}\left[|a|\|u\|_{p, M}^{p}+|k|\|u\|_{p, \partial M}^{p}\right],
$$

SO

$$
G_{q, r}(u) \geq\left(\lambda_{f, K, \eta, q, r}-1\right)\left[|a|\|u\|_{p, M}^{p}+|k|\|u\|_{p, \partial M}^{p}\right],
$$

write $\min (|a|,|k|)\left(\lambda_{f, K, \eta, q, r}-1\right)=\alpha_{\eta}+\beta_{\eta}$ such that $\frac{\alpha_{\eta}(|a|+|k|)}{\beta}=\frac{A+B}{K_{1}+K_{2}}$, where $A, B, K_{1}, K_{2}$ are the constants appearing in the Sobolev and trace Sobolev inequalities. Then

$$
\begin{aligned}
G_{q, r}(u) \geq & \alpha_{\eta}\left(\|u\|_{p, M}^{p}+\|u\|_{p, \partial M}^{p}\right)+\frac{\beta}{|a|+|k|}\left[-G_{q, r}(u)+\left\|\nabla_{g} u\right\|_{p, M}^{p}\right. \\
& \left.+\frac{p}{q} \int_{M}\left|f^{-}\right||u|^{q} d v_{g}+\frac{p}{r} \int_{\partial M}\left|K^{-}\right||u|^{r} d \sigma_{g}\right]
\end{aligned}
$$


that is

$$
\begin{aligned}
\left(1+\frac{\beta_{\eta}}{|a|+|k|}\right) G_{q, r}(u) \geq & \frac{\beta_{\eta}}{|a|+|k|} \\
& {\left[\left\|\nabla_{g} u\right\|_{p, M}^{p}+\frac{\alpha_{\eta}(|a|+|k|)}{\beta_{\eta}}\left(\|u\|_{p, M}^{p}+\|u\|_{p, \partial M}^{p}\right)\right] . }
\end{aligned}
$$

Then, by the Sobolev and trace Sobolev inequalities we get

$$
\begin{aligned}
\left(1+\frac{\beta_{\eta}}{|a|+|k|}\right) G_{q, r}(u) & \geq \frac{\beta_{\eta}}{|a|+|k|}\left[\left\|\nabla_{g} u\right\|_{p, M}^{p}+\frac{A+B}{K_{1}+K_{2}}\right. \\
& \geq \frac{\left.\left(\|u\|_{p, M}^{p}+\|u\|_{p, \partial M}^{p}\right)\right]}{(|a|+|k|)\left(K_{1}+K_{2}\right)}\left(\ell^{\frac{p}{q}}+\ell^{\frac{p}{r}}\right) .
\end{aligned}
$$

Thus

$$
G_{q, r}(u) \geq \frac{\beta_{\eta}}{\left(K_{1}+K_{2}\right)\left(|a|+|k|+\beta_{\eta}\right)}\left(\ell^{\frac{p}{q}}+\ell^{\frac{p}{r}}\right) .
$$

On the other hand, we have

$$
\begin{aligned}
E_{q, r}(u) & =G_{q, r}(u)-\frac{p}{q} \int_{M} f^{+}|u|^{q} d v_{g}-\frac{p}{r} \int_{\partial M} K^{+}|u|^{r} d \sigma \\
& \geq G_{q, r}(u)-\left(\frac{p}{q} \sup _{M} f+\frac{p}{r} \sup _{\partial M} K\right) \ell \\
& \geq s\left(\ell^{\frac{p}{q}}+\ell^{\frac{p}{r}}\right)-\left(\frac{p}{q} \sup _{M} f+\frac{p}{r} \sup _{\partial M} K\right) \ell
\end{aligned}
$$

where

$$
s=\min \left(1, \frac{\beta_{\eta}}{\left(K_{1}+K_{2}\right)\left(|a|+|k|+\beta_{\eta}\right)}\right) .
$$

Now, the function

$$
g: \ell \longrightarrow \frac{s}{2}\left(\ell^{\frac{p}{q}}+\ell^{\frac{p}{r}}\right)-\left(\sup _{M} f+\sup _{\partial M} K\right) \ell,
$$

attains a positive maximum in the interval $] 0, \ell_{2, q, r}\left[\right.$ where $g\left(\ell_{2, q, r}\right)=0$. Make hypotheses on the functions $f$ and $K$ to assure that $g\left(\ell_{q, r}\right)>0$, where $\ell_{q, r}$ is defined by (5.1). Recall that $\ell_{1, q, r}>1$, then we have

$$
\begin{aligned}
g\left(\ell_{1, q, r}\right) \geq \ell_{1, q, r} & {\left[s \operatorname { m i n } \left(\left(\frac{\eta \int_{M}\left|f^{-}\right| d v_{g}}{1+|a| \operatorname{vol}(M)^{1-\frac{p}{q}}}\right)^{\frac{q}{r}},\right.\right.} \\
& \left.\left.\left(\frac{\eta \int_{\partial M}\left|K^{-}\right| d v_{g}}{1+|k| \operatorname{vol}(\partial M)^{1-\frac{p}{r}}}\right)^{\frac{q r-p q}{r^{2}-p q}}\right)-\left(\sup _{M} f+\sup _{\partial M} K\right)\right],
\end{aligned}
$$


so if we require that

$$
\begin{aligned}
& \sup _{M} f+\sup _{\partial M} K \leq C_{q, r} \\
& \quad=\frac{s}{2} \min \left(\left(\frac{\eta \int_{M}\left|f^{-}\right| d v_{g}}{1+|a| \operatorname{vol}(M)^{1-\frac{p}{q}}}\right)^{\frac{q}{r}},\left(\frac{\eta \int_{\partial M}\left|K^{-}\right| d v_{g}}{1+|k| \operatorname{vol}(\partial M)^{1-\frac{p}{r}}}\right)^{\frac{q^{r}-p q}{r^{2}-p q}}\right),
\end{aligned}
$$

we get $g\left(\ell_{1, q, r}\right)>0$ and then $\ell_{1, q, r}<\ell_{2, q, r}$. Thus if $\ell \in\left[\ell_{1, q, r}, \ell_{2, q, r}\right]$, then

$$
\mu_{\ell, q, r}=E_{q, r}(u)>\frac{s}{2}\left(\ell^{\frac{p}{q}}+\ell^{\frac{p}{r}}\right)>0,
$$

and therefore $\mu_{\ell, q, r}>0$ for all $\ell \in\left[\ell_{1, q, r}, \ell_{2, q, r}\right]$.

Moreover, put $\ell_{1}=\lim _{(q, r) \rightarrow\left(p^{*}, p^{* *}\right)} \ell_{1, q, r}, \ell_{2}=\lim _{(q, r) \rightarrow\left(p^{*}, p^{* *}\right)} \ell_{2, q, r}$ and suppose that $\ell_{1}=\ell_{2}$, by continuity of the function $g$ above and by (5.3) we get

$$
\begin{aligned}
0 & =\frac{s}{2}\left(\ell_{1}^{\frac{-p}{n}}+\ell_{1}^{\frac{1-p}{n-1}}\right)-\left(\sup _{M} f+\sup _{\partial M} K\right) \\
& \geq \frac{s}{2} \ell_{1}^{\frac{1-p}{n}},
\end{aligned}
$$

thus $\ell_{2}>\ell_{1}$ and it is clear by (5.4) that the $\operatorname{limit}_{(q, r) \rightarrow\left(p^{*}, p^{* *}\right)} \mu_{\ell, q, r}$ do not go to zero.

In what follows, by mean of the Mountain Pass Theorem, we show that the existence of the interval $\left[\ell_{1}, \ell_{2}\right]$ leads to the existence of a second critical point of the functional $E_{q, r}$. First, we prove the following lemma

Lemma 5.7. The Plais-Smale condition is satisfied for the functional $E_{q, r}$, $q<p^{*}$ and $r<p^{* *}$.

Proof. First, we claim that each Plais-Smale sequence for the functional $E_{q, r}$ is bounded in $H_{1}^{p}(M)$. In fact, let $u_{n} \in H_{1}^{p}(M)$ be a sequence such that $E_{q, r}\left(u_{n}\right) \underset{n \rightarrow \infty}{\rightarrow} \gamma$ and $E_{q, r}^{\prime}\left(u_{n}\right) \underset{n \rightarrow \infty}{\rightarrow} 0$, then we have

$$
\begin{aligned}
E_{q, r}\left(u_{n}\right)-\frac{1}{q} E_{q, r}^{\prime}\left(u_{n}\right) u_{n}= & \left(1-\frac{p}{q}\right)\left[\left\|\nabla_{g} u_{n}\right\|_{p, M}^{p}+a\left\|u_{n}\right\|_{p, M}^{p}+k\left\|u_{n}\right\|_{p, M}^{p}\right] \\
& -\left(\frac{1}{r}-\frac{p}{q}\right) \int_{\partial M} K\left|u_{n}\right|^{r} d \sigma_{g},
\end{aligned}
$$

and

$E_{q, r}\left(u_{n}\right)-\frac{1}{p} E_{q, r}^{\prime}\left(u_{n}\right) u_{n}=\left(1-\frac{p}{q}\right) \int_{M} f\left|u_{n}\right|^{q} d v_{g}+\left(1-\frac{p}{r}\right) \int_{\partial M} K\left|u_{n}\right|^{r} d \sigma_{g}$,

so, for every $\varepsilon>0$, there exists $n_{o}$ such that for all $n \geq n_{o}$, we have

$$
\left.\left|\left(1-\frac{p}{q}\right) \int_{M} f\right| u_{n}\right|^{q} d v_{g}+\left(1-\frac{p}{q}\right) \int_{\partial M} K\left|u_{n}\right|^{r} d \sigma_{g}-\gamma \mid \leq \varepsilon+o\left(\left\|u_{n}\right\|_{H_{1}^{p}(M)}\right),
$$

and

$$
\begin{gathered}
\left.\left|\left(1-\frac{p}{q}\right)\left[\left\|\nabla_{g} u_{n}\right\|_{p, M}^{p}+a\left\|u_{n}\right\|_{p, M}^{p}+k\left\|u_{n}\right\|_{p, M}^{p}\right]-\left(\frac{1}{r}-\frac{p}{r}\right) \int_{\partial M} K\right| u_{n}\right|^{r} d \sigma_{g}-\gamma \mid \\
\leq \varepsilon+o\left(\left\|u_{n}\right\|_{H_{1}^{p}(M)}\right) .
\end{gathered}
$$


Let $\ell>0$ be such that $\mu_{\ell, q, r}>0$ and put $v_{n}=\beta_{n} u_{n}$, where $\beta_{n}>0$ is such that $\beta_{n}^{q}\|u\|_{q, M}^{q}+\beta_{n}^{r}\|u\|_{r, M}^{r}=\ell$. We observe that $v_{n}$ is bounded in $L_{q}(M)$ and $L_{r}(\partial M)$.

Now, we have

$$
\begin{aligned}
& \left.\left|\frac{1}{\beta_{n}^{q}}\left(1-\frac{p}{q}\right) \int_{M} f\right| v_{n}\right|^{q} d v_{g}+\frac{1}{\beta_{n}^{r}}\left(1-\frac{p}{r}\right) \int_{\partial M} K\left|v_{n}\right|^{r} d \sigma_{g}-\gamma \mid \\
& \quad \leq \varepsilon+o\left\|v_{n}\right\|_{H_{1}^{p}(M)},
\end{aligned}
$$

and

$$
\begin{aligned}
& \mid \frac{1}{\beta_{n}^{p}}\left(1-\frac{p}{q}\right)\left[\left\|\nabla_{g} v_{n}\right\|_{p, M}^{p}+a\left\|v_{n}\right\|_{p, M}^{p}+k\left\|v_{n}\right\|_{p, M}^{p}\right] \\
& \quad-\frac{1}{\beta_{n}^{r}}\left(\frac{1}{r}-\frac{p}{q}\right) \int_{\partial M} K\left|v_{n}\right|^{r} d \sigma_{g}-\gamma \mid \leq \varepsilon+o\left\|v_{n}\right\|_{H_{1}^{p}(M) .}
\end{aligned}
$$

Since the sequence $v_{n}$ is bounded in $L_{q}(M)$ and $L_{r}(\partial M)$, then by $(6.3)$ it is bounded in $H_{1}^{p}(M)$. Moreover, we affirm that the sequence $u_{n}$ is bounded in $L_{q}(M)$ and $L_{r}(\partial M)$. In fact, if the sequence $u_{n}$ goes to infinity in $L_{q}(M)$ or $L_{r}(\partial M)$ then the sequence $\beta_{n}$ goes to zero when $n$ goes to infinity. This implies by mean of inequalities (6.2) and (6.3) that $E_{q, r}\left(v_{n}\right) \rightarrow 0$ as $n \rightarrow \infty$.

Since $v_{n} \in \mathcal{S}_{\ell, m, q, r}$, then $E_{q, r}\left(v_{n}\right) \geq \mu_{\ell, q, r}>0$, this is a patent contradiction. Hence, $u_{n}$ is bounded in $L_{q}(M)$ and $L_{r}(\partial M)$ and since $E_{q, r}^{\prime}\left(u_{n}\right) u_{n} \rightarrow 0$ then, $\left\{u_{n}\right\}_{n \in \mathbb{N}}$ is bounded in $H_{1}^{p}(M)$.

Thus, up to a subsequence $u_{n}$ converges to a function $u$ weakly in $H_{1}^{p}(M)$ and strongly in $L_{q}(M), L_{p}(M), L_{r}(\partial M)$ and $L_{p}(\partial M)$. By Brezis-Lieb [14] lemma, we obtain

$$
\begin{aligned}
\left\|\nabla_{g}\left(u_{n}-u\right)\right\|_{p, M} \leq & |a|\left\|u_{n}-u\right\|_{p, M}^{p}+|k|\left\|u_{n}-u\right\|_{p, \partial M}^{p}+\sup _{M} f\left\|u_{n}-u\right\|_{q, M}^{q} \\
& +\sup _{\partial M} K\left\|u_{n}-u\right\|_{r, \partial M}^{r}+o(1) \\
\leq & o(1) .
\end{aligned}
$$

which means that the subsequence $u_{n}$ converges strongly to $u$ in $H_{1}^{p}(M)$.

Now, we are in position to prove Theorem 3.2.

Proof of Theorem 3.2.

\section{- Existence of first solution}

Let $\ell_{1}>0$ be such that $\mu_{\ell_{1}, q, r}=0$, the curve $\ell \rightarrow \mu_{\ell, q, r}$ is negative for $\ell \in] 0, \ell_{1}[$.

Set

$$
\mu_{\ell_{q, r}, q, r}=\inf _{\mathcal{D}_{\ell, q, r}} E_{q, r}
$$

where

$$
\mathcal{D}_{\ell, q, r}=\left\{u \in H_{1}^{p}(M): u>0,\|u\|_{q, M}^{q}+\|u\|_{r, \partial M}^{r} \leq \ell, \ell<\ell_{1}\right\} .
$$


Take $\ell$ as small as $\mu_{\ell, q, r}<0$, by lemma (5.1) there exists $u \in H_{1}^{p}(M)$ such that $\|u\|_{q, M}^{q}+\|u\|_{r, \partial M}^{r}=\ell$ and $E_{q, r}(u)=\mu_{\ell, q, r}$ in such way that, $u \in \mathcal{D}_{\ell, q, r}$ and

$$
\mu_{\ell_{q, r}, q, r} \leq E_{q, r}(u)=\mu_{\ell, q, r}<0 .
$$

By using the well known Ekeland Variational Principle, in the set $\mathcal{D}_{\ell, q, r}$ we can find a sequence $\left\{u_{q, r, n}\right\}_{n}$ such that $E_{q, r}\left(u_{q, r, n}\right) \underset{n \rightarrow \infty}{\rightarrow} \mu_{\ell_{q, r}, q, r}$ and $E_{q, r}^{\prime}\left(u_{q, r, n}\right) \underset{n \rightarrow \infty}{\rightarrow} 0$. Obviously, the sequence $u_{q, r, n}$ is bounded in $H_{1}^{p}(M)$, then up to a subsequence, $u_{q, r, n}$ converges to a function $u_{q, r}$ weakly in $H_{1}^{p}(M)$ and strongly in $L_{s}(M), L_{t}(\partial M)$ for $s<p^{*}$ and $t<p^{* *}$, almost everywhere on $M$ and in the sense of trace on $\partial M$. Thus, $u_{q, r} \in \mathcal{D}_{\ell, q, r}$ and

$$
E_{q, r}\left(u_{q, r}\right) \leq \lim _{n \rightarrow \infty} \inf E_{q, r}\left(u_{q, r, n}\right)=\mu_{\ell_{q, r}, q, r}<0 .
$$

Furthermore, by the weak convergence in $H_{1}^{p}(M), L_{\frac{q}{q-1}}(M)$ and $L_{\frac{r}{r-1}}(\partial M)$, it follows that for every $v \in H_{1}^{p}(M)$

$$
\left\langle E^{\prime}\left(u_{q, r}\right), v\right\rangle=\lim _{n \rightarrow \infty}\left\langle E^{\prime}\left(u_{q, r, n}\right), v\right\rangle=0 .
$$

On the other hand, since for almost every where in $M$ we have $\left|\nabla_{g} u\right|=\left|\nabla_{g}\right| u||$ for all $u \in H_{1}^{p}(M)$, we get $E_{q, r}\left(u_{q, r, n}\right)=E_{q, r}\left(\left|u_{q, r, n}\right|\right)$ and $E_{q, r}^{\prime}\left(\left|u_{q, r, n}\right|\right) \rightarrow 0$, which gives together with (5.7) and (5.8) that $u_{q, r}$ is positive critical point of $E_{q, r}$ with $E_{q, r}\left(u_{q, r}\right)<0$.

\section{- Existence of second solution}

Now, we prove that there exists a second critical point $v_{q, r}$ with $E_{q, r}\left(v_{q, r}\right)>0$.

Let $\ell_{1}$ and $\ell_{2}$ be such that

$$
\begin{aligned}
& \mu_{\ell_{1}, q, r}=E_{q, r}\left(u_{\ell_{1}}\right)=0, \\
& \mu_{\ell_{2}, q, r}=E_{q, r}\left(u_{\ell_{2}}\right)=0,
\end{aligned}
$$

and consider

$$
\mu_{q, r}=\inf _{g \in \Gamma} \max _{s \in[0,1]} E_{q, r}(g(s))
$$

where

$$
\Gamma=\left\{g \in \mathcal{C}\left([0,1], H_{1}^{p}(M)\right): g(0)=u_{\ell_{1}}, g(1)=u_{\ell_{2}}\right\} .
$$

We claim that $\mu_{q, r}$ is a critical value of the functional $E_{q, r}$. In fact, if it is not, then there exists $\varepsilon>0$ small such that $E_{q, r}$ does not possess any critical value in the interval $\left[\mu_{q, r}-\varepsilon, \mu_{q, r}+\varepsilon\right]$. Thus, by the deformations Lemma we can find a function $\phi_{t}: H_{1}^{p}(M) \longrightarrow H_{1}^{p}(M), t \in[0,1]$, continuous in $t$ such that:

1. $\phi_{0}(u)=u, \forall u \in H_{1}^{p}(M)$,

2. $\phi_{t}(u)=u, \forall u \in H_{1}^{p}(M)$ such that $E_{q, r}(u) \notin\left[\mu_{q, r}-\varepsilon, \mu_{q, r}+\varepsilon\right]$,

3. $\forall u \in H_{1}^{p}(M)$, s.t $E_{q, r}(u) \leq \mu_{q, r}+\varepsilon$, then $E_{q, r}\left(\phi_{1}(u)\right) \leq \mu_{q, r}-\varepsilon$.

Now, let $g \in \Gamma$ be such that $\max _{s \in[0,1]} E_{q, r}(g(s)) \leq \mu_{q, r}+\varepsilon$. By definition of $u_{\ell_{1}}, u_{\ell_{2}}$ and property (2) of the function $\phi_{t}$, we have

$$
\begin{aligned}
& \phi_{t}\left(u_{\ell_{1}}\right)=u_{\ell_{1}}, \\
& \phi_{t}\left(u_{\ell_{2}}\right)=u_{\ell_{2}} .
\end{aligned}
$$


In particular, the curve $\phi_{1}(g) \in \Gamma$ which gives that $\mu_{q, r} \leq \max _{s \in[0,1]} E_{q, r}$ $\left(\phi_{1}(g(s))\right)$, but by property (3), we have that $\max _{s \in[0,1]} E_{q, r}\left(\phi_{1}(g(s))\right) \leq \mu_{q, r}$ $-\varepsilon$, which makes a contradiction. $\mu_{q, r}$ is then a critical level for the functional $E_{q, r}$ and

$$
\mu_{q, r}>\sup _{\ell \in\left[\ell_{1}, \ell_{2}\right]} \mu_{\ell, q, r}>0 .
$$

Therefore, there exists $v_{q, r}>0$ a critical point of $E_{q, r}$ with $E_{q, r}\left(v_{q, r}\right)=\mu_{q, r}$ $>0$ and Theorem 3.2 is proven.

\section{Critical problem: multiplicity result}

In this section, we prove existence of at least two positive solutions of the problem (1.1). We will study the limits of the sequences $u_{q, r}$ and $v_{q, r}$ as $(q, r)$ goes to $\left(p^{*}, p^{* *}\right)$. Besides the non-compactness of the inclusions $H_{1}^{p}(M) \hookrightarrow L_{p^{*}}(M)$ and $L_{p^{* *}}(\partial M)$, we face serious difficulties in proving that the sequences converge towards different limits and this fact is due essentially to the nature of the functions $f$ and $K$ that change sign. Here, the curve $\ell \rightarrow \mu_{\ell, q, r}$ will play an important role in overcoming these problems.

Let $E$ be the functional

$$
\begin{aligned}
E= & \int_{M}\left|\nabla_{g} u\right|^{p} d v_{g}+a \int_{M}|u|^{p} d v_{g}+k \int_{\partial M}|u|^{p} d \sigma_{g} \\
& -\frac{n-p}{n} \int_{M} f|u|^{p^{*}} d v_{g}-\frac{n-p}{n-1} \int_{\partial M} K|u|^{p^{* *}} d \sigma_{g} .
\end{aligned}
$$

\subsection{Existence of first solution}

Proof of Theorem 3.1. For $(q, r) \in] p, p^{*}[\times] p, p^{* *}\left[\right.$, let $u_{q, r}>0$ be the the positive critical point of the functional $E_{q, r}$ such that $E_{q, r}\left(u_{q, r}\right)<0$. First we claim that the sequence $u_{q, r}$ is bounded in $H_{1}^{p}(M)$ with respect to $(q, r)$. In fact, by construction of $u_{q, r}$ we have

$$
\begin{aligned}
& \left\|u_{q, r}\right\|_{q, M}^{q}+\left\|u_{q, r}\right\|_{r, \partial M}^{r}=\ell_{q, r}<\ell_{1} \\
& \quad<\max \left(\left(\frac{2 v o l(M)^{1-\frac{p}{n}}|a|}{\eta \int_{M}\left|f^{-}\right| d v_{g}}\right)^{\frac{n}{p}},\left(\frac{2 v o l(\partial M)^{1-\frac{p}{n-1}}|k|}{\eta \int_{\partial M}\left|K^{-}\right| d \sigma_{g}}\right)^{\frac{n-1}{p}}\right),
\end{aligned}
$$

then $u_{q, r}$ is bounded in $L_{q}(M)$ and $L_{r}(\partial M)$. Moreover, by (5.7) we have

$$
\begin{gathered}
\left\|\nabla_{g} u_{q, r}\right\|_{p, M} \leq \mu_{r, q}+|a| \operatorname{vol}(M)^{\frac{p}{n}} \ell_{1}^{\frac{n-p}{n}}+|k| \operatorname{vol}(\partial M)^{\frac{p-1}{n-1}} \ell_{1}^{\frac{n-p}{n-1}} \\
+\left(\sup _{M} f+\sup _{\partial M} K\right) \ell_{1},
\end{gathered}
$$

this implies, since $\mu_{\ell, q, r}<0$, that the sequence $u_{q, r}$ is bounded in $H_{1}^{p}(M)$. Thus, we can obtain a subsequence $u_{q, r}$ and a function $u \in H_{1}^{p}(M)$ such that

1. $u_{q, r}$ converges weakly to $u$ in $H_{1}^{p}(M)$

2. $u_{q, r}$ converges strongly to $u$ in $L_{p}(M), L_{p}(\partial M), L_{p^{*}-1}(M)$ and $L_{p^{* *}-1}$ $(\partial M)$ 
3. $u_{q, r}$ converges almost everywhere to $u$ in $M$ and in the sense of trace on $\partial M$

4. The sequence $\nabla_{g} u_{q, r}$ converges almost everywhere to $\nabla_{g} u$

Thus, $u \geq 0$ is critical point of $E$ and by Brezis-Lieb lemma [14] together with Sobolev and trace Sobolev inequalities, we get

$$
\begin{aligned}
E_{q, r}\left(u_{q, r}\right)-E_{q, r}(u)= & \left\|\nabla_{g}\left(u_{q, r}-u\right)\right\|_{p, M}^{p}-\left(1-\frac{p}{q}\right) \int_{M} f\left|u_{q, r}-u\right|^{q} d v_{g} \\
& -\left(1-\frac{p}{q}\right) \int_{\partial M} K\left|u_{q, r}-u\right|^{r} d \sigma_{g}+o(1) \\
\geq & \frac{1}{K_{1}+K_{2}}\left[\left\|u_{q, r}-u\right\|_{q, M}^{p}+\left\|u_{q, r}-u\right\|_{r, M}^{p}\right] \\
& -\sup _{M} f\left\|u_{q, r}-u\right\|_{q, M}^{q}-\sup _{\partial M} K\left\|u_{q, r}-u\right\|_{r, \partial M}^{r}+o(1)
\end{aligned}
$$

then, taking into account that $\lim _{(q, r) \rightarrow\left(p^{*}, p^{* *}\right)}\left\|u_{q, r}-u\right\|_{q, M}^{q}+\left\|u_{q, r}-u\right\|_{r, \partial M}^{r} \leq$ $\ell, \ell>0$, by Brezis-Lieb Lemma again, we get

$$
\begin{aligned}
E(u) \leq & \lim _{(q, r) \rightarrow\left(p^{*}, p^{* *}\right)} E_{q, r}\left(u_{q, r}\right)-\frac{1}{K_{1}+K_{2}} \lim _{(q, r) \rightarrow\left(p^{*}, p^{* *}\right)}\left[\left\|u_{q, r}-u\right\|_{q, M}^{p}\right. \\
& \left.+\left\|u_{q, r}-u\right\|_{r, M}^{p}\right]+\left(\sup _{M} f+\sup _{\partial M} K\right) \ell .
\end{aligned}
$$

Sine, a priori, we have that $\lim _{(q, r) \rightarrow\left(p^{*}, p^{* *}\right)}\left[\left\|u_{q, r}-u\right\|_{q, M}^{p}+\left\|u_{q, r}-u\right\|_{r, M}^{p}\right]>$ 0 , by taking $\ell$ small enough, we obtain $E(u)<0$, thus $u>0$ and we are done.

\subsection{Existence of second solution}

Now, we consider the sequence $\left\{v_{q, r}\right\}$ of positive solutions of the subcritical problem (3.1) obtained by the Mountain Pass Theorem. We prove that $\left\{v_{q, r}\right\}$ will converge to a non zero different critical point of $E$. We recall that the sequence $\left\{v_{q, r}\right\}$ fulfills the following properties

$$
E_{q, r}^{\prime}\left(v_{q, r}\right)=0 \text { and } E_{q, r}\left(v_{q, r}\right)=\mu_{q, r}>0 .
$$

First, we prove the following lemma

Lemma 6.1. The sequence of functions $v_{q, r}$ is bounded in $H_{1}^{p}(M)$.

Proof. Let $\ell_{1}, \ell_{2}, 0<\ell_{1}<\ell_{2}$ be two real numbers with the associated functions $u_{\ell_{1}}$ and $u_{\ell_{1}}$ such that

$$
\begin{aligned}
& \mu_{\ell_{1}, q, r}=E_{q, r}\left(u_{\ell_{1}}\right)=0 \\
& \mu_{\ell_{2}, q, r}=E_{q, r}\left(u_{\ell_{2}}\right)=0 .
\end{aligned}
$$

For $s \in[0,1]$, let $g$ be the curve $g(s)=s u_{\ell_{1}}+(1-s) u_{\ell_{2}}$. Then by definition of $\mu_{q, r}$ we have

$$
\mu_{q, r} \leq \max _{s \in[0,1]} E_{q, r}(g(s))
$$


Since $E_{q, r}(g(0))=E_{q, r}(g(1))=0$ together with the fact that we can find $s^{\prime} \in(0,1)$ such that

$$
\left\|g\left(s^{\prime}\right)\right\|_{q, M}^{q}+\left\|g\left(s^{\prime}\right)\right\|_{r, \partial M}^{r}=\frac{\ell_{1}+\ell_{2}}{2} \in\left(\ell_{1}, \ell_{2}\right)
$$

that is $E_{q, r}\left(g\left(s^{\prime}\right)\right)>\mu_{\frac{\ell_{1}+\ell_{2}}{2}, q, r}>0$, it follows that the curve $s \longrightarrow E_{q, r}(g(s))$ attains for a certain $s_{o} \in(0,1)$ a positive maximum. Thus

$$
\begin{aligned}
\mu_{q, r} \leq E_{q, r}\left(g\left(s_{o}\right)\right) & =\left(1-\frac{p}{q}\right) \int_{M} f\left|g\left(s_{o}\right)\right|^{q} d v_{g}+\left(1-\frac{p}{r}\right) \int_{\partial M} K\left|g\left(s_{o}\right)\right|^{r} d \sigma_{g} \\
& \leq\left(\sup _{M} f+\sup _{\partial M} K\right)\left(\ell_{1}+\ell_{2}\right) .
\end{aligned}
$$

which gives that the sequence $\mu_{q, r}$ is uniformly bounded in $(q, r)$.

Now, it remains to show that the sequence $v_{q, r}$ is bounded in $L_{q}(M)$ and $L_{r}(\partial M)$. We proceed similarly to the proof of Theorem 3.2. Let $\ell_{0} \in\left(\ell_{1}, \ell_{2}\right)$ be such that $\mu_{\ell_{o}, q, r}=\sup _{\ell} \mu_{\ell, q, r}>0$ and consider the sequence $w_{q, r}=\beta_{q, r} v_{q, r}$ where $\beta_{q, r}>0$ is such that

$$
\beta_{q, r}^{q}\left\|v_{q, r}\right\|_{q, M}^{q}+\beta_{q, r}^{r}\left\|v_{q, r}\right\|_{r, \partial M}^{r}=\ell_{o}
$$

The sequence $w_{q, r}$, such as defined, satisfies

$$
\mu_{q, r}=\left(1-\frac{p}{q}\right) \beta_{q, r}^{-q} \int_{M} f w_{q, r}^{q} d v_{g}+\left(1-\frac{p}{r}\right) \beta_{q, r}^{-r} \int_{\partial M} K w_{q, r}^{r} d \sigma_{g}
$$

and

$$
\begin{aligned}
E_{q, r}\left(w_{q, r}\right)= & \beta_{q, r}^{p} \mu_{q, r}+\frac{p}{q}\left(\beta_{q, r}^{p-q}-1\right) \int_{M} f\left|w_{q, r}\right|^{q} d v_{g} \\
& +\frac{p}{r}\left(\beta_{q, r}^{p-r}-1\right) \int_{\partial M} K\left|w_{q, r}\right|^{r} d \sigma_{g} .
\end{aligned}
$$

Suppose by contradiction that the sequence $v_{q, r}$ goes to infinity in $L_{q}(M)$ and $L_{r}(\partial M)$ as $(q, r)$ goes to $\left(p^{*}, p^{* *}\right)$. Then, by $(6.2)$ the sequence $\beta_{q, r}$ should go to zero. Since $\mu_{q, r}$ is bounded, we get necessarily by (6.3) that $\int_{M} f\left|w_{q, r}\right|^{q} d v_{g}$ and $\int_{\partial M} K\left|w_{q, r}\right|^{r} d \sigma_{g}$ do go to zero as $(q, r)$ goes to $\left(p^{*}, p^{* *}\right)$ and $\beta_{q, r}^{-q} \int_{M} f\left|w_{q, r}\right|^{q} d v_{g}, \beta_{q, r}^{-r} \int_{\partial M} K\left|w_{q, r}\right|^{r} d \sigma_{g}$ are both bounded. Thus, by (6.4), we get that $E_{q, r}\left(w_{q, r}\right)$ goes to zero as $(q, r)$ goes to $\left(p^{*}, p^{* *}\right)$.

On the other hand, we have $E_{q, r}\left(w_{q, r}\right)>\mu_{\ell_{o}, q, r}$ and by Lemma 5.6 the sequence $\mu_{\ell_{0}, q, r}$ does not go to zero as $(q, r)$ goes to $\left(p^{*}, p^{* *}\right)$, this makes a contradiction. Therefore, the sequence $v_{q, r}$ is bounded in $L_{q}\left(M\right.$ and $L_{r}(\partial M)$ and since it satisfies

$$
\begin{aligned}
\left\|\nabla_{g} v_{q, r}\right\|_{p, M}^{p}= & \mu_{q, r}+|a|\left\|v_{q, r}\right\|_{p, M}^{p}+|k|\left\|v_{q, r}\right\|_{p, \partial M}^{p} \\
& +\int_{M} f\left|v_{q, r}\right|^{q} d v_{g}+\int_{\partial M} K\left|u_{q, r}\right|^{r} d \sigma_{g},
\end{aligned}
$$

then it is bounded in $H_{1}^{p}(M)$.

Now that the sequence $v_{q, r}$ is bounded in $H_{1}^{p}(M)$, we can extract a subsequence that converges strongly to a function $v \geq 0$ in $L_{p}(M)$ and $L_{p}(\partial M)$ and weakly in $H_{1}^{p}(M), L_{\frac{p^{*}}{p^{*}-1}}(M)$ and $L_{\frac{p^{* *}}{p^{* *}-1}}(\partial M)$. The function $v \geq 0$ is then a 
critical point of the functional $E$. But, this is not enough to conclude existence of second solution because in spite of the fact that $\lim _{(q, r) \rightarrow\left(p^{*}, p^{* *}\right)} E_{q, r}\left(v_{q, r}\right)$ $-E_{q, r}\left(u_{q, r}\right)>0$, we could have $v=u$ or $v=0$ regarding the lack of the strong convergence of the sequence $v_{q, r}$ to $v$ in $L_{p^{*}}(M)$ and $L_{p^{* *}}(\partial M)$. In the following lemmas, we give sufficient conditions to prevent such cases from occurring.

First, note that the first solution $u$ satisfies that $\|u\|_{p^{*}, M}^{p^{*}}+\|u\|_{p^{* *}, \partial M}^{p^{* *}} \leq$ $\ell<\ell_{1}$. Take $\ell$ such that

$$
\ell<\frac{\left(\sup _{M} f\right)^{-\frac{n-p}{p}} K_{1}^{-n}}{\left|\inf _{M} f\right|+\left|\inf _{\partial M} K\right|}
$$

we get then

$$
E(u)+\frac{p}{n}\left(\sup _{M} f\right)^{1-\frac{n}{p}} K_{1}^{-n}>0 .
$$

Let us prove the following lemma

Lemma 6.2. Suppose that the sequence $v_{q, r}$ converges strongly to the function $u$ in $L_{p}(M)$ and $L_{p}(\partial M)$ and that the functions $f$ and $K$ satisfy

$$
\frac{n(p-1)}{p(n-1)}\left(\sup _{M} f\right)^{\frac{n-p}{p}}\left(\sup _{\partial M} K\right)^{-\frac{n-p}{p-1}} K_{1}^{n} K_{2}^{-\frac{p(n-1)}{p-1}} \geq 1 .
$$

If the following condition is satisfied

$$
\lim _{(q, r) \rightarrow\left(p^{*}, p^{* *}\right)} \mu_{q, r}<E(u)+\frac{p}{n}\left(\sup _{M} f\right)^{1-\frac{n}{p}} K_{1}^{-n}
$$

then the sequence $v_{q, r}$ converges strongly to $u$ in $H_{1}^{p}(M)$.

Proof. By lemma (6.1) the sequence $v_{q, r}$ is bounded. We may assume that $v_{q, r}$ converges to $u$ weakly in $H_{1}^{p}(M)$, almost every where on $M$ and in the sense of trace on $\partial M$.

In particular, we assume that the sequences of measures $\left|\nabla_{g} v_{q, r}\right|^{p} d v_{g},\left|v_{q, r}\right|^{p^{*}}$ $d v_{g}$ and $\left|v_{q, r}\right|^{*^{* *}} d \sigma_{g}$ converge weakly in the sense of measures respectively to bounded nonnegative measures $d \mu, d \nu$ and $d \pi$.

Thus, by a version of a concentration-compactness theorem for manifolds with boundary [12], there exist at most countable index set $I$, sequence of points $\left\{x_{i}\right\}_{i \in I} \subset \bar{M}$ and positive numbers $\left\{\mu_{i}\right\}_{i \in I},\left\{\nu_{i}\right\}_{i \in I},\left\{\pi_{i}\right\}_{i \in I}$ such that

$$
\begin{aligned}
& d \mu \geq\left|\nabla_{g} u\right|^{p} d v_{g}+\sum_{i \in I} \mu_{i} \delta_{x_{i}}, \\
& d \nu=|u|^{p^{*}} d v_{g}+\sum_{i \in I} \nu_{i} \delta_{x_{i}}, \text { and } \\
& d \pi=|u|^{p^{* *}} d \sigma_{g}+\sum_{i \in J} \pi_{i} \delta_{x_{i}} .
\end{aligned}
$$

Moreover,

$$
\nu_{i}^{\frac{1}{p^{*}}} \leq K_{1} \mu_{i}^{\frac{1}{p}} \text { and } \pi_{i}^{\frac{1}{p^{* *}}} \leq K_{2} \mu_{i}^{\frac{1}{p}}
$$


Take $x_{i} \in \bar{M}$ in the support of the singular part of $\mu, \nu$ and $\pi$ and for $\varepsilon>0$, let $\phi$ be a $\mathcal{C}^{\infty}\left(B\left(x_{i}, \varepsilon\right)\right)$ cut-off function such that supp $\phi \subset\left(B\left(x_{i}, \varepsilon\right), \phi \equiv 1\right.$ on $\left(B\left(x_{i}, \frac{\varepsilon}{2}\right)\right)$ and $\left|\nabla_{g} \phi\right| \leq C$. Then, we get

$$
\begin{aligned}
\int_{B\left(x_{i}, \varepsilon\right)} \phi d \mu= & \lim _{(q, r) \rightarrow\left(p^{*}, p^{* *}\right)} \int_{B\left(x_{i}, \varepsilon\right)}\left|\nabla_{g} v_{q, r}^{p-2}\right| g\left(\nabla_{g} v_{q, r}, \nabla_{g} \phi\right) d v_{g} \\
= & -a \int_{B\left(x_{i}, \varepsilon\right)}|u|^{p-2} u \phi d v_{g}-k \int_{B\left(x_{i}, \varepsilon\right)}|u|^{p-2} u \phi d \sigma_{g} \\
& +\int_{B\left(x_{i}, \varepsilon\right)} f \phi d \nu+\int_{B\left(x_{i}, \varepsilon\right)} K \phi d \pi
\end{aligned}
$$

this implies, after letting $\varepsilon$ tend to zero, that for each $i \in J$, depending on whether $x_{i} \in M$ or $x_{i} \in \partial M$,

$$
\mu_{i} \leq f\left(x_{i}\right) \nu_{i}, \mu_{i} \leq K\left(x_{i}\right) \pi_{i} .
$$

In particular, we assume that either $f\left(x_{i}\right)>0$ or $K\left(x_{i}\right)>0$, because otherwise we get $\mu_{i}=\nu_{i}=\pi_{i}=0$ and we are done.

Now, suppose that there exists $i_{o} \in I$ such that $\nu_{i_{o}} \neq 0$ or $\pi_{i_{o}} \neq 0$ (depending on whether $x_{i_{o}}$ is in $M$ or $\partial M$ ), then we get

$$
\begin{aligned}
\lim _{\left(q, r \rightarrow\left(p^{*}, p^{* *}\right)\right.} \mu_{q, r} & =\lim _{\left(q, r \rightarrow\left(p^{*}, p^{* *}\right)\right.}\left(\left(1-\frac{p}{q}\right) \int_{M} f v_{q, r}^{q} d v_{g}+\left(1-\frac{p}{r}\right) \int_{\partial M} K v_{q, r}^{r} d \sigma_{g}\right) \\
& =\frac{p}{n} \int_{M} f u^{p^{*}} d v_{g}+\frac{p-1}{n-1} \int_{\partial M} K u^{p^{* *}} d \sigma_{g}+\frac{p}{n} f\left(x_{i_{o}}\right) \nu_{i}+\frac{p-1}{n-1} K\left(x_{i_{o}}\right) \pi_{i} \\
& \geq E(u)+\max \left(\frac{p}{n}\left[\sup _{M} f\right]^{1-\frac{n}{p}} K_{1}^{-n}, \frac{p-1}{n-1}\left[\sup _{M} K\right]^{1-\frac{n-1}{p-1}} K_{2}^{\frac{-p(n-1)}{p-1}}\right),
\end{aligned}
$$

this implies by (6.5), (6.7) and (6.8) that

$$
\lim _{\left(q, r \rightarrow\left(p^{*}, p^{* *}\right)\right.} \mu_{q, r} \geq E(u)+\frac{p}{n}\left[\sup _{M} f\right]^{1-\frac{n}{p}} K_{1}^{-n},
$$

which contradicts the hypothesis of the lemma. Thus, we get $\mu_{i}=\nu_{i}=\pi_{i}=0$ and the sequence $v_{q, r}$ converges strongly to $u$ in $L_{p^{*}}(M)$ and $L_{p^{* *}}(\partial M)$.

In the following, we give a sufficient condition in order to get satisfied condition (6.6) of Lemma 6.2.

Lemma 6.3. Suppose that there exists a positive function $\Phi \in H_{1}^{p}(M)$ such that $I(\Phi)>0, \int_{M} f \Phi^{p^{*}} d v_{g}>0, \int_{\partial M} K \Phi^{p^{* *}} d \sigma_{g}>0$ and

$$
0<\sup _{\lambda \in[0,1]} E(\lambda \Phi)<E(u)+\frac{p}{n}\left[\sup _{M} f\right]^{1-\frac{n}{p}} K_{1}^{-n}
$$

then condition (6.6) of Lemma 6.2 is satisfied.

Proof. Let $u_{\ell_{1}}, u_{\ell_{2}}$ be such that $E_{q, r}\left(u_{\ell_{1}}\right)=E_{q, r}\left(u_{\ell_{2}}\right)=0$ and $\ell_{1}=\left\|u_{\ell_{1}}\right\|_{q, M}^{q}+$ $\left\|u_{\ell_{1}}\right\|_{r, \partial M}^{r}<\ell_{o}<\ell_{2}=\left\|u_{\ell_{2}}\right\|_{q, M}^{q}+\left\|u_{\ell_{2}}\right\|_{r, \partial M}^{r}$, where $\ell_{o}$ is such that $\mu_{\ell_{o}}=$ $\sup _{\ell \in] \ell_{1}, \ell_{2}[} \mu_{q, r, \ell}$. Then, the curve $\lambda \rightarrow E_{q, r}\left(\frac{\alpha \lambda-1}{\alpha-1} u_{\ell_{2}}\right), \alpha>1, \lambda \in\left[\frac{1}{\alpha}, \infty[\right.$, starts from zero, increases towards a positive maximum for $\lambda<1$ and then decreases to minus infinity. 
Now, suppose that there exists a function $\Phi \in H_{1}^{p}(M)$ such that $\|\Phi\|_{q, M}^{q}+$ $\|\Phi\|_{r, \partial M}^{r}>\ell_{2}$. Let $\delta_{o}>\delta_{1}>1$ be two constants and consider the curve $\lambda \rightarrow E_{q, r}\left(\left(1-\delta_{o} \lambda\right)\left(\delta_{1} \lambda-1\right) \Phi\right), \lambda \in\left[\frac{1}{\delta_{1}}, \infty\right]$, then we have,

$$
\begin{gathered}
\frac{d E_{q, r}\left(\left(1-\delta_{o} \lambda\right)\left(\delta_{1} \lambda-1\right) \Phi\right)}{d \lambda}=p\left(\delta_{1}+\delta_{o}\right)\left[\left|1-\delta_{o} \lambda \| \delta_{1} \lambda-1\right|\right]^{p-1}\left(1-\frac{2 \delta_{o} \delta_{1}}{\delta_{1}+\delta_{o}} \lambda\right) \\
{\left[\left\|\nabla_{g} \Phi\right\|_{p, M}^{p}+a\|\Phi\|_{p, M}^{p}+k\|\Phi\|_{p, \partial M}^{p}-\left(\left|1-\delta_{o} \lambda \| \delta_{1} \lambda-1\right|\right)^{q-p} \int_{M} f \Phi^{q} d v_{g}\right.} \\
\left.-\left(\left|1-\delta_{o} \lambda \| \delta_{1} \lambda-1\right|\right)^{r-p} \int_{\partial M} K \Phi^{r} d v_{g}\right] .
\end{gathered}
$$

Let us denote by $F_{\Phi}(\lambda)$ the function

$$
\begin{aligned}
F_{\Phi}(\lambda)= & I(\phi)-\left(\left(1-\delta_{o} \lambda\right)\left(\delta_{1} \lambda-1\right)\right)^{q-p} \int_{M} f \Phi^{q} d v_{g} \\
& -\left(\left(1-\delta_{o} \lambda\right)\left(\delta_{1} \lambda-1\right)\right)^{r-p} \int_{\partial M} K \Phi^{r} d \sigma_{g}
\end{aligned}
$$

with $\lambda \in\left[\frac{1}{\delta_{o}}, \frac{1}{\delta_{1}}\right], I(\phi)>0, \int_{M} f \Phi^{q} d v_{g}>0$ and $\int_{\partial M} K \Phi^{r} d \sigma_{g}>0$.

Then, in the interval $\left(\frac{1}{\delta_{o}}, \frac{1}{\delta_{1}}\right)$, there exist at most two values $\frac{1}{\delta_{2}}>\frac{\delta_{o}+\delta_{1}}{2 \delta_{o} \delta_{1}}>$ $\frac{1}{\delta_{3}}$ of $\lambda$ such that $F_{\Phi}\left(\frac{1}{\delta_{2}}\right)=F_{\Phi}\left(\frac{1}{\delta_{3}}\right)=0, F_{\Phi}(\lambda)>0, \lambda \in\left(\frac{1}{\delta_{o}}, \frac{1}{\delta_{3}}\right) \cup\left(\frac{1}{\delta_{2}}, \frac{1}{\delta_{1}}\right)$ and $F_{\Phi}(\lambda)<0, \lambda \in\left(\frac{1}{\delta_{3}}, \frac{1}{\delta_{2}}\right)$.

Hence, there exists $\delta_{o}>\delta_{o}^{\prime}>\delta_{1}$ such that the curve $\lambda \rightarrow E_{q, r}((1-$ $\left.\left.\delta_{o} \lambda\right)\left(\delta_{1} \lambda-1\right) \Phi\right)$ is positive for $\lambda \in\left[\frac{1}{\delta_{o}^{\prime}}, \frac{1}{\delta_{1}}\right]$ and attains positive maximum at $\lambda_{o}=\frac{1}{\delta_{2}}$.

On the other hand, the curve $\lambda \rightarrow E_{q, r}\left(\left(1-\delta_{o} \lambda\right) u_{\ell_{1}}\right)$, starts from zero, decreases to negative minimum in the interval $\left(0, \frac{1}{\delta_{o}}\right)$ and then increases to infinity. Now, take $\delta_{o}$ close to $\delta_{o}^{\prime}$ and $\delta_{1}$ as close to 1 as

$$
E_{q, r}\left(\frac{\delta_{1} \lambda-1}{\delta_{1}-1} u_{\ell_{1}}\right)<\sup _{\lambda \in\left(\frac{1}{\delta_{o}^{\prime}}, \frac{1}{\delta_{1}}\right)} E_{q, r}\left(\left(1-\delta_{o} \lambda\right)\left(\delta_{1} \lambda-1\right) \Phi\right)=E_{q, r}\left(\frac{1}{\delta_{2}} \Phi\right)
$$

Consider the curve

$$
g(\lambda)= \begin{cases}\left(1-\delta_{o} \lambda\right) u_{\ell_{1}}, & 0 \leq \lambda \leq \frac{1}{\delta_{o}} \\ \left(1-\delta_{o} \lambda\right)\left(\delta_{1} \lambda-1\right) \Phi, & \frac{1}{\delta_{o}} \leq \lambda \leq \frac{1}{\delta_{1}} \\ \frac{\delta_{1} \lambda-1}{\delta_{1}-1} u_{\ell_{2}}, & \frac{1}{\delta_{1}} \leq \lambda \leq 1 .\end{cases}
$$

Suppose that the condition of the lemma is satisfied, then in a neighborhood $V_{\left(p^{*}, p^{* *}\right)}$ of $\left(p^{*}, p^{* *}\right)$ we can assume for every $(q, r) \in V_{\left(p^{*}, p^{* *}\right)}$ that

$$
0<\sup _{\lambda \in[0,1]} E_{q, r}(\lambda \Phi)<E_{q, r}(u)+\frac{p}{n}\left[\sup _{M} f\right]^{1-\frac{n}{p}} K_{1}^{-n}
$$


then

$$
\begin{aligned}
\mu_{q, r} & \leq \sup _{\lambda \in[o, 1]} E_{q, r}(g(\lambda)) \leq E_{q, r}\left(\frac{1}{4 \delta_{o}} \Phi\right) \\
& \leq E_{q, r}(u)+\frac{p}{n}\left[\sup _{M} f\right]^{1-\frac{n}{p}} K_{1}^{-n} .
\end{aligned}
$$

As a result, we obtain the following lemma

Lemma 6.4. Suppose that the functions $f$ and $K$ satisfy

$$
\frac{p(n-1)}{n(p-1)}\left(\sup _{M} f\right)^{-\frac{n-p}{p}}\left(\sup _{\partial M} K\right)^{\frac{n-p}{p-1}} K_{1}^{-n} K_{2}^{\frac{p(n-1)}{p-1}} \leq 1
$$

and that there exists a function $\Phi \in H_{1}^{p}(M)$ with $I(\Phi)>0, \int_{M} f \Phi^{p^{*}} d v_{g}>0$, $\int_{\partial M} K \Phi^{p^{* *}} d \sigma_{g}>0$ such that

$$
0<\sup _{\lambda \in[0,1]} E(\lambda \Phi)<E(u)+\frac{p}{n}\left[\sup _{M} f\right]^{1-\frac{n}{p}} K_{1}^{-n}
$$

Then none of the following situations $\lim _{(q, r) \rightarrow\left(p^{*}, p^{* *}\right)} v_{q, r}=0$ or

$$
\lim \left\|v_{q, r}-u\right\|_{p, M}^{p}=\lim _{(q, r) \rightarrow\left(p^{*} p^{* *}\right)}\left\|v_{q, r}-u\right\|_{p, \partial M}^{p}=0
$$

can occur.

Proof. If the sequence $v_{q, r}$ converges to zero function as $(q, r)$ goes to $\left(p^{*}, p^{* *}\right)$, we can repeat the proof of lemma 6.2 without the term $E(u)$ we get

$$
\lim _{(q, r) \rightarrow\left(p^{*}, p^{* *}\right)} \mu_{q, r} \geq \frac{p}{n}\left[\sup _{M} f\right]^{1-\frac{n}{p}} K_{1}^{-n}
$$

Since we have $E(u)<0$, under the hypothesis of the lemma we get by Lemmas 6.2 and 6.3 a contradiction, that is, $v_{q, r}$ does not converge to zero function as $(q, r)$ goes to $\left(p^{*}, p^{* *}\right)$ and at the same time the sequence $v_{q, r}$ can not satisfy that

$$
\lim \left\|v_{q, r}-u\right\|_{p, M}^{p}=\lim _{(q, r) \rightarrow\left(p^{*} p^{* *}\right)}\left\|v_{q, r}-u\right\|_{p, \partial M}^{p}=0
$$

since $E_{q, r}\left(v_{q, r}\right)=\lim _{(q, r) \rightarrow\left(p^{*}, p^{* *}\right.} \mu_{q, r}>0$.

Now, the proof of theorem 3.3 follows

Proof of Theorem 3.3. By Theorem 3.1 there exists a solution $u$ of problem (1.1) with $E(u)>0$. Let $v_{q, r}$ the sequence of solutions of the subcritical problem (3.1) given by theorem (3.2). By Lemma 6.1 the sequence $v_{q, r}$ is bounded in $H_{1}^{p}(M)$, then after passing to a subsequence we assume that $v_{q, r}$ converges, when $(q, r)$ goes to $\left(p^{*}, p^{* *}\right)$ to a function $v$ weakly in $H_{1}^{p}(M)$, strongly in $L_{p}(M)$ and $L_{p}(\partial M)$, almost everywhere in $M$ and in the sense of trace on $\partial M$ and it converges to $v^{p^{*}-1}$ weakly in $L_{\frac{p^{*}}{p^{*}-1}}(M)$ and to $v^{p^{* *}-1}$ weakly in $L_{\frac{p^{* *}}{p^{* *}-1}}(\partial M)$. Then we get that $v$ is a weak solution of problem (1.1). 
Under hypothesis of the theorem we get by Lemmas $6.1,6.2,6.3$ and 6.4 that $v \neq 0$ and $v \neq u$, that is problem (1.1) admits a second weak solution.

\section{Acknowledgments}

I would like to express deep gratitude to Prof. Boumedien Abdellaoui for providing me with the paper [1] and for his valuable discussion about the manuscript.

\section{References}

[1] Abdellaoui, B., Peral, I.: On quasilinear elliptic equations related to some Caffarelli-Khon-Nirenberg inequalities. Commun. Pure Appl. Anal. 2(4), 539-566 (2003)

[2] Adams, R.A., Fournier, J.J.F.: Sobolev Spaces, 2nd edn. Academic Press (2003)

[3] Ambrosetti, A., Li, Y.Y., Malchiodi, A.: Scalar curvature under boundary conditions. C.R.A.S. Paris, t. 330, Série 1, pp. 1013-1018 (2000)

[4] Ambrosetti, A., Li, Y.Y., Malchiodi, A.: Yamabe and scalar curvature problems under boundary conditions. Math. Ann. 322, 667-699 (2002)

[5] Aubin, T.: Some Nonlinear Problems in Riemannian Geometry. Springer, New York (1998)

[6] Aubin, T., Cotsiolis, A.: Equations non linéaires avec le $p$-laplacien et la fonction exponentielle sur les variétés Riemanniennnes compactes. Bull. Sci. Math. 124, 1-19 (2000)

[7] Benalili, M.: Existence and multiplicity of solutions to elliptic equations of fourth order on compact manifolds. Dyn. PDE 6(3), 203-225 (2009)

[8] Benalili, M., Maliki, Y.: Generalized scalar curvature type equation on complete Riemannian manifolds. Electron. J. Differ. Equ. 146, 1-18 (2004)

[9] Benalili, M., Maliki, Y.: Solving p-Laplacian on complete Riemannian manifolds. Electron. J. Differ. Equ. 155, 1-9 (2006)

[10] Benalili, M., Maliki, Y.: Generalized prescribed scalar curvature type equation on compact manifolds of negative scalar curvature. Rocky Mt. J. Math. 5(37), 1399-1413 (2007)

[11] Benalili, M., Maliki, Y.: Multiplicity of solutions for elliptic quasilinear equations with critical exponent on compact manifolds. Nonlinear Anal. 71, 5946$5960(2009)$

[12] Biezuner, R.J.: Best constants in Sobolev trace inequalities. Nonlinear Anal. 54, 575-589 (2003)

[13] Biezuner, R.J.: Best constants, optimal Sobolev inequalities on Riemannian manifolds and applications. Thesis, University of New Jersey (2003) 
[14] Brezis, H., Lieb, E.H.: A relation between pointwise convergence of functions and functionals. Proc. AMS 88, 486-490 (1983)

[15] Cherrier, P.: Problémes de Neumann non linéaires sur les variétés Riemanniennes. J. Funct. Anal. 57(2), 154-206 (1984)

[16] Cherrier, P.: Cas d'exception du théoréme d'inclusion de Sobolev sur les variétés Riemanniennes et applications. Bull. Sci. Math. 105, 235-288 (1981)

[17] Cherrier, P.: Meilleurs constantes dans des inégalités relatives aux espaces de Sobolev. Bull. Sci. Math. 108, 225-262 (1984)

[18] Cotsiolis, A., Labropoulos, N.: A Neumann problem with the $q$-laplacian on a solid torus in the critical of supercritical case. Electron. J. Differ. Equ. 164, $1-18(2007)$

[19] Cotsiolis, A., Labropoulos, N.: Best constants in Sobolev inequalities on manifolds with boundary in the presence of symmetries and applications. Bull. Sci. Math. 132, 562-574 (2008)

[20] Dajdli, Z., Malchiodi, A., Ahmedou, M.O.: The prescribed boundary mean curvature problem on $\mathbb{B}^{4}$. J. Differ. Equ. 206, 373-398 (2004)

[21] Druet, O.: Generalized scalar curvature type equations on compact Riemannian manifolds. Proc. R. Soc. Edinburgh A 130, 269-289 (2000)

[22] Escobar, J.: Conformal deformation of a Riemannian metric to scalar flat metric with constant mean curvature on the boundary. Ann. Math. 136, 1-50 (1992); 139, 749-750 (1994)

[23] Escobar, J.: The Yamabe probelm on manifolds with boundary. J. Differ. Geom. 35, 21-84 (1992)

[24] IL'Yasov, Y., Runst, T.: Positive solutions for indefinite inhomogenous Neumann elliptic problems. Electron. J. Differ. Equ. 57, 1-21 (2003)

[25] Lieberman, G.M.: Boundary regularity for solutions of degenerate elliptic equations. Nonlinear Anal. TMA 12, 1203-1219 (1988)

[26] Pohozaev, S.I.: On an approach to nonlinear equations. Doklady Acad. Sci. USSR 247, 1327-1331 (1979)

[27] Rauzy, A.: Courbures scalaires des variétés d'invariant conforme négatif. Trans. AMS 374(12), 4729-4745 (1995)

Youssef Maliki

Department of Mathematics

University Aboubekr Belkaïd of Tlemcen

Tlemcen

Algeria

e-mail: malyouc@yahoo.fr

Received: 18 October 2011.

Accepted: 18 December 2011. 